\title{
Teaching Assessment of Engineering Education by Information and Communication Technologies
}

\author{
Yi ZHANG \\ School of Energy and Mechanical Engineering, Nanjing Normal University, Nanjing, 210042, China \\ email: zhy.68@163.com
}

\begin{abstract}
Keywords: Engineering Education; Teaching Assessment; Information and Communication Technologies
\end{abstract}

\begin{abstract}
A well-designed teaching assessment strategy can motivate students, and help teachers to develop deep learning. Recent learning theories lay stress on the value of dialogue, negotiation and feedback of teaching assessment. Teaching assessment provides the feedback and shared meanings essential to teacher and student. Information and communication technologies can facilitate the best aspects of teaching assessment. This paper shows how information and communication technologies support teaching assessment of engineering education.
\end{abstract}

\section{Introduction}

Teaching assessment has long been an essential component of teaching and learning. From the student's view, teaching assessment is often central to the interpretation of the aims, goals and focus of teaching process. Formative assessment and feedback contribute to learning through reflection and interpretation and are valued as a diagnostic tool to identify misconceptions of the course [1]. Summative assessment provides a measure of personal and comparative progress within a teaching team[2]. End-point examinations are valued as a symbol of pass of the course [3].

For teachers, teaching assessment is a key element of the teaching process, providing a mechanism for feedback and means to encourage the students to practice of skills and techniques. Traditional grading and feedback are time-consuming activities and it needs at least one teacher to grade one assignment.

The traditional forms of teaching assessment, whether in the form of reports or examinations, are expensive to implement. Nevertheless, they are the basis of teaching assessment. Information and communication technologies are a powerful and an efficient teaching assessment method suitable for education institutions.

\section{Execution of Teaching Assessment}

The last two decades have seen great efforts to understand the processes of teaching and learning in engineering education. One fruitful approach has been to distinguish between approaches of learning of different students. Research has identified a range of student behaviors [4]. The surface learning is characterized by a concentration on facts, a reliance on memory and a reluctance (or inability) to engage with the fuller understanding, which is, on the contrary, often referred to as 'deep learning'. More significant, however, for the design of appropriate teaching assessment, is the observation that many students adopt a correct approach of learning.

Students identify the behaviors consciously or subconsciously that will gain them high grades, and direct their activities to such ends. Student learning research has repeatedly demonstrated the impact of teaching assessment on students' approaches to learning. For example, if the teacher asks them to understand the physics and chemistry of muscle contraction, but test them on the names of the muscles, and they will 'learn' the names but not be able to explain how contraction happens. If the students are asked to understand narrative perspective in the novel but are tested on the author's background and they will know a lot about the author and little about narrative perspective.[5] 


\section{Application of Information and Communication Technologies}

The explosion of information and communication technologies has presented teachers with the opportunity to review the whole question of teaching assessment and to explore new forms of deliverables that support and nurture students' creativity. When information and communication technologies are applied to teaching assessment, the multi-method characteristic enabled by computer technology expands the range of channels available to students to demonstrate their understanding of the course. For example, instead of using narrowly defined learning outcomes tested by examinations, information and communication technologies offer a total environment where real life skills, such as written and verbal communication, collaboration and team work can be assessed by giving the students multiple channels of expression, such as visualization and multimedia.

Information and communication technologies can change the quality of the learning experience, and can be used to create authentic environments for teaching assessment. There are four broad groups of information and communication technologies classified according to the complexity of the technology deployed. The challenge of application of information and communication technologies to teaching assessment is to match learning outcomes of the students with appropriate kind of information and communication technologies.

\section{Transfer Function of Information and Communication Technologies}

Information and communication technologies can either transfer assessment results, such as an online quiz, or to transfer the resources associated with an assessment, which might include the background documents, World Wide Web links, or software.

Many of the earliest examples of such function are of the 'objective test' format, also referred to as computer-assisted assessment and computer-based assessment, constructed using optical card readers and HTML forms. User's responses typically take the form of: selecting one or more answers from a list; matching options from two or more lists; checking a box or radio button; short or long answers using input boxes and text-areas, respectively. Information and communication technologies underlying this function are relatively cheap to develop, built as the technologies are on web browsers and web servers.

The online quiz format, typically completed by an individual student, offers little opportunity for student to negotiate or social interact with each other. However, reformulating the assignment as a collaborative activity can facilitate constructivist learning theory elements. Access to assignments or examinations may be both time-released and time-limited.

The greatest challenge, and hence cost, of developing computer-assisted assessment function is the creation of the bank of questions from which individual assignments are built. Historically, this function has been factual questions targeted at the lower-level skills, but as the tools become better understood, so it is expected to develop formative and summative computer-assisted assessment function under the direction of the cognitive learning theory.

\section{Execute Function of Information and Communication Technologies}

Information and communication technologies can provide an integral element of learning, but for which the teaching assessment is related to the deliverables students create with these tools. Alternative tools include simulations and simulators, engineering design and evaluation tools, WWW and CD-based resource repositories, mind-mapping tools and electronic portfolios. A subset tool specifically designed to facilitate communication and group work is also included in this function.

One of the early examples of these tools was SPICE, a simulator for the design of analogue and digital circuits based on the prediction of linear and non-linear components. SPICE has gained wide acceptance across the electronics industry and commercial products have extended its range of features. 
Other engineering disciplines have made extensive use of products such as Autodesk's AutoCAD and the various add-ons for structural and dynamic analysis and three-dimensional visualization. Assignments built around these products would require students to undertake a design activity and submit the circuit-diagram, drawing, or visualization along with their report.

The high development cost of single-model simulators has encouraged teachers to explore the use of constructive tools, such as MatLab, Mathematica, MathCad and Octave, which build upon powerful numerical and symbolic analysis engines coupled with graphical user interfaces. MatLab, for example, offers a rich set of toolboxes covering many facets of engineering, such as process simulation, curve fitting, image and signal processing, telecommunications simulation and neural networks. The advantage of these general-purpose tools is that they can be employed throughout an entire study program, building upon student skills and extending teaching assessment to the higher skill levels of the cognitive learning theory.

The ubiquitous spreadsheet has proved a powerful tool for both experimentation and teaching assessment. At one level, students operate on a completed model and investigate the effects of varying different parameters, while at another level students adapt the model itself. But it was found this tool was invaluable in teaching the principles of engineering disciplines and other aspects of engineering disciplines.

\section{Organize Function of Information and Communication Technologies}

The organize function of information and communication technologies can provide an organizing or unifying framework that underpins formative and summative assessment. These frameworks are commonly referred to as virtual learning environments, learning management systems and learning content management systems.

At one end of the function are single course products, which can be a self-contained virtual learning environments. These products can be developed to allow the creation of study environments which can exploit material in a wide range of media and allow easy provision of mechanisms for support, feedback, collaboration and self-assessment without recourse by staff to skills such as scripting and mark-up languages.

At the other end of the function are the large-scale tools such as Blackboard's Learning System6 and WebCT's Campus Edition7 and the University of Twente's TeleTOP. These tools are aimed at supporting students, faculty, instructional designers and administrators.

The key features of these tools include an teaching assessment area where individual assignments are posted for students to download. A repository for resources is also included, which are usually in the forms of documents, URIs, or streamed audio and video. A calendar serves for study and assignments with warnings given as a submission date approaches. A repository can give the aims, objectives, outcomes and competencies of the course. A tracking system can summarize completed assignments, complete with historical grading data. A repository can store the feedback from the teacher. Announcements and access lead to the public and private computer-mediated communication areas

All these tools offer a wide range of features and facilities that can be tailored to the requirements of individual courses or institutions.

\section{Assess Function of Information and Communication Technologies}

The final function included in the above tools should support or undertake assignment grading and may provide some feedback for formative and summative assessment.

First within this function are the complimentary parts of the computer-aided assessment function described previously. Automated grading is the essential characteristic of this function, which is essentially a binary decision based on logical comparison of numeric values or keywords and phrases. An HTML form returns the index of the option selected and is compared to a stored answer. Feedback is provided in the form of prepared comments based on further logical comparisons of the returned value. In most cases this teaching assessment process is totally unaware of the cognitive processes of the student. So a system danger lies in the 
assessment process.

Computer adaptive testing tools aim to compensate for the variability in students' ability. As testing proceeds an iterative estimate of a student's ability is made and the difficulty of the next question selected is based on this estimate. According to proponents, computer adaptive testing tools provide a better indicator of a student's ability because the final score includes a measure of the difficulty of the questions answered, rather than the total number of correct answers.

Tools that are targeted at automated grading of essays, reports and projects, and built on developments in natural language processing is by far the most challenging tools to project. The most notable examples are Project Essay Grader, E-Rater and Intelligent Essay Assessor. It is reported that E-Rater achieved agreement with human rater scores of between 87 and $94 \%$.

\section{Conclusion}

Teaching assessment is not simply a measure of student performance, it is often central to a student's understanding of the aims and outcomes of a course, and its primary purpose should be to enhance learning. What observed by teaching assessment is a merging between formative assessment based on information and communication technologies and technology support for learning where frequent formative assessment can promote active learning.

Students and teachers will require support moving from pen and paper-based assessment systems to those based on information and communication technologies. For students the key issue is gaining confidence in the use of the technology. For teachers the issues relate to changing workloads, quality assurance and careful evaluation of systems. The benefits of application teaching assessment based on information and communication technologies will be acquired in the form of stimulating and challenging environments for learning.

\section{Acknowledgement}

This study was sponsored by the Teaching Reform Study Project of Nanjing Normal University (Project No. 18122000090491).

\section{References}

[1] SAMBELL, K., SAMBELL, A. and SEXTON, G. Student perceptions of the learning benefits of computer-assisted assessment: a case study in electronic engineering. In S. BROWN, J. BULL and P. RACE (eds), Computer Assisted Assessment in Higher Education (London: Kogan Page), 1999, 179-191.

[2] RYAN, S., SCOTT, B., FREEMAN, H. and PATEL, D. The Virtual University: The Internet and Resource-based Learning (London: Kogan Page) 2000.

[3] WELLER, M. Delivering Learning on the Net (London: Kogan Page), 2000.

[4] RAMSDEN, P. Learning to Teach in Higher Education (London: Routledge) ,1992.

[5] BIGGS, J. B. Aligning the curriculum to promote good learning. Presented at Constructive Alignment in Action: Imaginative Curriculum Symposium, Learning and Teaching Support, Network Generic Centre, November, 2002. 